\title{
Die Ethik, die ich meine
}

\section{Markus Gnädinger}

Research fellow der Einheit für Hausarztmedizin (EHAM) der Universität Zürich

\begin{abstract}
Das ischs Lied vo de Studie, wo gäng scho abgschlosse sy, bevor sie ume hey agfange. D'ökter wüssid nid, wa mache, u die Chranke müe waarte! (frei nach Mani Matter)
\end{abstract}

\section{Zusammenfassung}

Die Anforderungen rechtlicher und organisatorischer Art an Studien sind hoch. Hoffnungsvolle Ansätze zu einer Forschungskultur werden gefährdet durch die enormen Ansprüche an die Studienhaftung. Durch das nun in Überarbeitung begriffene Humanforschungsgesetz könnten die Probleme verschärft und perpetuiert werden.

Es ist höchste Zeit, dass der Gesetzgeber etablierte medizinische Verfahren und normale Risiken des Alltags explizit aus der Studienhaftung ausschliesst.

\section{Unsichere Rechtslage}

Seit meinem letzten Unkenruf zur Frage der Studienhaftpflicht resp. Probandenversicherung (SÄZ 85: 317, 2004) ist nun einige Zeit ins Land gegangen. Damals ging es um eine grössere Studie zur Primärprävention von Frakturen mittels Gabe von 100000 E Vit. $D_{3}$ oder Placebo alle drei Monate für zwei Jahre. Die Studie sollte je 1000 Pensionäre von Alterspflegeheimen umfassen und musste wegen exorbitanter Gebühren von Fr. 70 000.- für die Probandenversicherung abgesagt werden, deren Abschluss von der Ethikkommission als unerlässlich angesehen worden war.

Seither hat sich im Bereich der nichtkommerziellen Forschung einiges getan. Die Schweizerische Akademie der medizinischen Wissenschaften (SAMW) hat Stipendien für hausärztliche Forscher* geäufnet. Ein neues Humanforschungsgesetz (HFG) ging im Frühling dieses Jahres in die Vernehmlassung und befindet sich nun in Überarbeitung. Ende März 2006 fand unter dem Patronat der SAMW in Bern eine Tagung interessierter Kreise zum neuen Gesetzesentwurf statt. Es ging darum, welche Forschungsaktivitäten Gegenstand des Gesetzes sein sollten (z.B. mit oder ohne Psychologen) und wie weit die Studienhaftung gehen sollte. Es war die Rede von «strenger» gegenüber «einfacher» Kausalhaftung.

\section{L'éthique telle que je la conçois}

Les exigences juridiques et d'organisation auxquelles doivent satisfaire les études scientifiques sont élevées. De bonnes prémisses à l'avènement d'une culture de la recherche sont mises en danger par les énormes attentes que l'on place dans les études en ce qui concerne la question de la responsabilité. La loi sur la recherche sur l'être humain, en cours de remaniement, devrait accentuer ce problème et le perpétuer.

Pour l'auteur du présent article, il est grand temps que le législateur exclue clairement les procédures médicales établies et les risques normaux du quotidien de la responsabilité dont doivent répondre les études.

Während bei letzterer die Möglichkeit eines Entlastungsbeweises für den Haftpflichtigen möglich ist, ist ein solcher bei der strengen Kausalhaftung (auch Gefährdungshaftung genannt) nicht möglich. Der HFG-Entwurf ist insoweit präzis, als nur Schäden im Zusammenhang mit der Studie der Haftung unterliegen. Nicht darunter fallen demzufolge solche aufgrund vorbestehender Krankheiten bzw. des normalen Krankheitsverlaufs sowie Schäden, die in keinerlei Kontext zur Studie stehen. Schwieriger wird es allerdings, wenn jemand auf dem Weg in die Praxis angefahren wird, oder wenn er in der Praxis stolpert und sich verletzt. Oder wie wäre es, wenn ein Proband während eines psychologischen Videointerviews einen Herzinfarkt bekommt?

Dieses Problem ist aber schon im geltenden Recht vorhanden - die Diskussion um das neue HFG hat das Problem nur verdeutlicht, nicht erzeugt. Da die Rechtslage unsicher ist, reisst sich die Privatassekuranz nicht darum, wissenschaftliche Studien versichern zu dürfen, und verlangt (im Zweifelsfall) lieber einen satten Aufschlag statt kostendeckender Gebühren. 
** Es geht mir keineswegs darum, den Ethikkommissionen (EK), die ihre wichtige Arbeit meist halbehrenamtlich leisten, eins «an Charre z'fahre», sondern darum, Probleme aufzuzeigen, welche die Durchführung wichtiger Studien behindern und letztlich auch die Arbeit in den EK erschweren.

\section{Aufgabe der Ethikkommissionen**}

Die kantonalen Ethikkommissionen (EK) haben die Aufgabe, zu überprüfen, ob eine geplante wissenschaftliche Studie die gesetzlichen Vorgaben erfüllt. Meist eckt eine Studie jedoch nicht im ethischen Bereich an, sondern in Fragen der Probandenaufklärung, des Datenschutzes oder der Haftung. So überrascht es nicht, dass die Pharmagiganten mit ihren beträchtlichen Ressourcen an Geld und Personal vor dem scharfen Auge der EK generell die «besseren» Gesuche abliefern, als dies «Hobbyforscher» mit nichtkommerziellen Projekten tun. Also hat eine unethische Studie, mit dem Zweck, mittels eines (an sich unnötigen) «Me-too-Präparates» einen beträchtlichen Teil des Marktes zu gewinnen, die grössere Chance, die EK $\mathrm{zu}$ passieren, ohne zerpflückt und zerzaust $\mathrm{zu}$ werden, als dies bei einer Hausarztstudie je der Fall sein kann, und sollte diese noch so ethisch sein. Eigentlich sollte man die EK deswegen gescheiter «Vorschriften-Einhaltungs-Überprüfungskommission» nennen. Das Verhältnis von Risiko und Nutzen fand eine starke Gewichtung im HFGEntwurf, wofür dieser aber einige Kritik einstecken musste!

\section{Die Probandenversicherung}

Eine kantonale Ärzteschaft möchte untersuchen, ob sich eine nächtliche Vortriagierung der Anrufe im Notfalldienst günstig auswirkt. Sie plant eine Studie und legt diese der EK vor. Die EK bewilligt den Versuch unter Auflagen. Es brauche a) ein schriftliches informiertes Einverständnis der Versuchsteilnehmer, b) eine öffentliche Bekanntmachung des Versuches in den Medien und c) den Nachweis, dass allfällige Haftungsansprüche aus dem Versuch gedeckt seien. Falls es für dieses Vorhaben eine Probandenversicherung bräuchte, wird es teuer! Man rechnet mit vier Anrufen pro Nacht und plant eine Studienphase von vier Monaten Länge; die Versicherung kostet 35 Franken (siehe oben): 4 Anrufe $\times 4$ Monate $\times 30$ Tage $\times$ Fr. 35.- = Fr. 16 800.-. Ein hübsches Sümmchen!

In unserer Praxis lassen wir die ErstjahresLehrtochter nur unter Aufsicht an unser «Mordinstrument» Nummer 1, das Praxistelefon! Da kann man die Bedenken der EK durchaus nachvollziehen. Andererseits hat das Callcenter in verschiedenen Publikationen gezeigt, dass es eine qualitativ hochstehende Arbeit leistet, die den Vergleich mit uns oft übernächtigten und genervten Notfallärzten kaum zu scheuen braucht. Und es geht beim geplanten Versuch ja nicht um ein «Heilmittel, invasive Verfahren oder Transplantate», so dass die Handhabe, eine Probandenversicherung zu verlangen, auch rechtlich nicht ganz hieb- und stichfest ist. Nun hat im genannten Fall der Haftpflichtversicherer des Callcenters eingelenkt und bestätigt, dass er in generöser Weise auch Risiken im Rahmen der geplanten Pilotstudie vergüten werde.

Insgesamt kann die Zusammenarbeit mit der EK also positiv bewertet werden. Dank kurzer Wege und weil man sich gegenseitig kennt, konnte der Entscheid der EK innert Monatsfrist erwirkt werden, ohne dass sich die Studie deswegen verzögert hätte. Dem Insistieren der EK, den Versuch in den Medien anzukündigen, kann leicht begegnet werden. Ebenso ist es eine kleine Sache, dem Patientenfragebogen ein Blatt mit dem «informierten Einverständnis» beizufügen. Der Preis von 400 Franken für den EK-Entscheid muss im Rahmen der Gesamtkosten als bescheiden angesehen werden. Wie wäre die Sache wohl ausgegangen, wenn man dafür eine überregionale oder gar eidgenössische EK hätte bemühen müssen?

Ein Wermutstropfen bleibt: Das Verlangen nach einer ausdrücklichen Haftungsdeckung für Schäden im Rahmen der Studie erscheint zwar legitim. Nur: Die Zusammenarbeit mit dem Callcenter ist eine spezielle Situation. Wollten wir eine «normale» Hausarztstudie versichern, wären normalerweise einerseits verschiedene Versicherer im Spiel und hätten diese andererseits nicht das gleiche Interesse an uns Praktikern, wie dies beim Grosskunden Callcenter der Fall ist. Es würde uns nichts anderes übrigbleiben, als entweder zu zahlen oder den Versuch abzublasen!

\section{Wenn zwei das gleiche tun, so tun sie nicht dasselbe}

So kommt es also darauf an, mit welcher Intention man irgendeine Handlung verrichtet. Wenn Sie irgendein klinisch anerkanntes Verfahren wählen, sei es diagnostischer, therapeutischer oder administrativer Art, so verfahren Sie innerhalb der üblichen Gleise ärztlicher Kunst. Wenn Sie Ihre Patientenkarten durchsehen und retrospektiv beurteilen, wie sich dieses oder jenes Verfahren bewährt hat - kein Problem, sofern Sie sich damit keine Schwierigkeiten mit Datenschutz resp. Arztgeheimnis einhandeln (das HFG sieht hier explizit die Verpflichtung vor, die Patienten auch für retrospektive Untersuchungen anzufragen, eine Versicherung ist dafür natürlich nicht nötig).

Wenn Sie Ihre Methodik aber standardisieren und ein bestimmtes Verfahren prospektiv auswerten möchten, so wird Ihr Projekt zur Studie. Nun gilt Ihr Streben nicht mehr nur der Heilung des Patienten, sondern es kommt der Wunsch nach Erkenntnisgewinn hinzu. Damit handeln Sie sich eine erhöhte Sorgfaltspflicht ein und müssen von Pontius bis Pilatus für die Eingabe bei der EK und die Probandenversicherung. Es versteht 
*** Es ist mir klar, dass es verschiedenste Gründe gibt, warum diese Vorstellung sich nicht so leicht in die Praxis umsetzen lässt. Es geht mir aber hier um eine längerfristige Zielvorstellung, die natürlich ins Medizinstudium und in die Gesetzgebung, aber vor allem in das Selbstverständnis von uns Ärzten hineindiffundieren sollte, damit längerfristig Veränderungen möglich sind. Es ist nämlich gar nicht so einfach zu definieren, wo die normale Klinik aufhört und Forschung anfängt. Wenn Sie in der Praxis Placebo einsetzen und den Fall dann publizieren; wenn Sie zwei gleichwertige therapeutische Optionen sehen und würfeln, welche Sie wählen wollen; wenn Sie Fallserien aufarbeiten, handelt es sich dann um Studien?

Eindeutig den «Rubikon» zur Forschung haben Sie aber überschritten, wenn Sie einem Patienten eine Massnahme angedeihen lassen, die ohne dessen Teilnahme an einem wissenschaftlichen Studienprotokoll nicht in Frage gekommen wäre. sich von selbst, dass solches dem Begehren der praktizierenden Ärzteschaft, sich in wissenschaftlichen Studien zu verwirklichen, nicht eben förderlich ist.

Seit einigen Jahren bin ich Mitglied der Kommission RRMA (der SAMW), die Gelder für hausärztliche Forschungsprojekte zu vergeben hat. Die Situation bei der Probandenversicherung und den Eingaben an die EK, deren Einwendungen bisweilen etwas «tüpflischiisserisch» empfunden werden, haben dazu geführt, dass hausärztliche Forschungsprojekte, so wie wir sie in der letzten Zeit zu beurteilen hatten, fast durchgehend als Untersuchungsobjekt uns Hausärzte selbst hatten und die Patienten aus dem Fokus ausklammerten - also «Nabelschauprojekte» waren, wie ich sie nenne, ohne dies despektierlich zu meinen. Die Ärzte als «Versuchskaninchen» müssen bis jetzt nämlich noch nicht versichert werden!

Kurzum, die einzigen, die in der Schweiz Forschung am Patienten machen können, sind neben den Spitalmedizinern die grossen Pharmaunternehmen. Und beforscht wird mithin nicht, was die Medizin benötigt, sondern was sich später mutmasslich wird verkaufen lassen.

\section{Die Lösung liegt im EJPD ...}

Die Lösung unseres Problems liegt (oder besser «hängt») im Büro unseres allseits geschätzten Justizministers. Christoph Blocher lässt sich bei Interviews gerne mit dem «Holzfäller» Ferdinand Hodlers ablichten. Wir brauchen eine solche kraftstrotzende Figur, die uns wie damals Alexander der Grosse den «gordischen Knoten» zerschlägt! Nun, die Federführung liegt im Fall des HFG beim BAG, zugehörig zu Pascal Couchepins Departement des Innern. Aber vielleicht findet sich dort auch ein passender «Holzfäller»!?!

Rings um die universitären hausärztlichen Einheiten, Institute, Instanzen, oder wie immer sie heissen, befinden sich Netzwerke forschungsinteressierter Hausärzte im Aufbau. Nun müssen endlich die Rahmenbedingungen für die klinische nichtkommerzielle Forschung den Notwendigkeiten und nicht die Forschungsprojekte den Gepflogenheiten der «Big Pharma» angepasst werden.

Damit dies funktionieren kann, müssen die Netzwerke sich um eine hochstehende Qualität ihrer Projekte bemühen, wobei hier die universitäre Verankerung helfen kann. So wird auch das Ansehen der «Bottom-up-Forschung» von uns Hausärzten steigen, und wir werden als Gesprächspartner, Wissens- und Erfahrungsquelle zunehmend wahr- und ernst genommen werden.

\section{Wunschliste}

Eine Möglichkeit wäre, die klinische Forschung zum integralen Bestandteil der ärztlichen Tätigkeit zu erklären, damit die Tatsache, dass jemand im Rahmen seiner Berufsausübung in einem Forschungsprojekt (mit)arbeitet, für sich allein genommen kein Grund für eine besondere Haftung mehr sein sollte.***

Dann sollten Projekte, die etablierte Verfahren gegeneinander abwägen, nicht der Studienhaftpflicht unterstellt sein. Oder anders gesagt, alle üblichen Methoden, die im Courant normal der Medizin Verwendung finden, dürfen nicht zu erweiterten Haftungsansprüchen führen, auch wenn sie in Studien angewendet und dokumentiert werden, solange der Patient das nach Meinung des Arztes für ihn bestmögliche Verfahren ausgerichtet erhält. Wichtig wäre dies vor allem in Fächern wie Onkologie, Orthopädie, HIV, wo sehr viele Patienten in Studien verfolgt werden.

Dasselbe gilt auch für normale Risiken des Alltags. Wenn jemand während einer Studie einen Zwischenfall erleidet, dessen Eintreten ohne die Studienteilnahme mutmasslich gleich wahrscheinlich gewesen wäre resp. der auch ohne die Teilnahme an der Studie eingetreten wäre, so soll dies durch die obligatorische Kranken- und Unfallversicherung und nicht durch die Studienhaftpflicht entschädigt werden. Dies ist übrigens im HFG auch so vorgesehen.

Für neue und unerprobte Verfahren ohne kommerziellen Hintergrund sollte eine Rahmenversicherung geschaffen werden, die z.B. bei der SAMW oder beim Kollegium für Hausarztmedizin (KHM) domiziliert werden könnte. Für die einzelnen Projekte müsste dann ein Anschlussvertrag abgeschlossen werden. Dies hätte verschiedene positive Auswirkungen: Der Versicherungsnehmer wäre dann ein Mitarbeiter von SAMW oder KHM mit wesentlich mehr diesbezüglicher Expertise als der hausärztliche Studienleiter und nicht so leicht zu übertölpeln wie dieser - und die Versicherung hätte ein deutlich gesteigertes Interesse an der pfleglichen Behandlung des Verhandlungspartners, da aus dieser Quelle ja ein kontinuierlicher Strom neuer Geschäfte resultieren würde.

\section{Zum Schluss}

Im HFG ist zwar für Versuche am Menschen eine strenge Kausalhaftung vorgeschlagen. Den Konflikt mit der nichtkommerziellen Forschung will man dort damit entschärfen, dass in der Verordnung des Bundesrates bei Versuchen mit urteilsfähigen Erwachsenen und nur minimalen Risi-ken von der Sicherstellungs- bzw. Versicherungspflicht abgesehen werden soll. Dies würde 
in der Tat unserer hausärztlichen Forschung sehr helfen.

Nehmen wir das Heft in die Hand! Das neue HFG wird uns, wenn einmal in Kraft gesetzt, Jahrzehnte erhalten bleiben. Werden wir jetzt aktiv, statt später darüber zu jammern! Klar, wir können in der Schweiz nicht ein «Sonderzüglein» fahren und sind bis zu einem gewissen Mass an internationale Vereinbarungen gebunden. Auch wenn das HFG an sich kein neues Recht setzen will, nur das bestehende klarer ausformulieren und ergänzen, und auch wenn es ein «Probandenschutz-» und nicht ein «Forschungsförderungsgesetz» sein will: Durch wenige klare Aussagen zur Haftung könnte die gegenwärtige Misere bei der Probandenversicherung deutlich gebessert werden.

Sonst gehen wir folgendem Szenario entgegen: Wenn durch entsprechende Vorschriften und eine engstirnige Auslegung derselben die nichtkommerzielle Forschung am Patienten in der Schweiz ausradiert worden ist, braucht es ja keine Ethikkommissionen mehr, das Problem hat dann von selbst eine überraschende Lösung gefunden! Alternativ könnten die EK ja dann sich selbst in Forschungsprojekten untersuchen, wie wir Hausärzte es jetzt schon tun ...

Düster wird's, das Spiel ist aus!

Wir alle gehn gar fromm nach Haus.

(frei nach W. Busch)

\section{Hemmen oder fördern Ethikkommissionen klinische Versuche?}

\section{Rainer Andenmatten \\ Dr. phil. II., Präsident der kantonalen Ethikkommission des Kantons Thurgau}

Korrespondenz:

Dr. phil. Rainer Andenmatten Kantonsapotheker

Postfach

CH-8596 Münsterlingen

rainer.andenmatten@stgag.ch
Obwohl das Stichwort «Deregulierung» politisch die Runde macht, nimmt im Heilmittelbereich die Regulierungsdichte stetig zu. Heilmittelgesetz und Verordnungen umfassen beim Stand 1. Oktober 2006 bereits 346 Seiten. Arbeitsweise der Ethikkommissionen und Anforderungen an klinische Versuche werden im HMG und HFG akribisch detailliert vorgegeben.

Diese Regelungsdichte einerseits und der Ruf der Pharmaindustrie nach möglichst einer einzigen zentralen Ethikkommission für alle Versuchsstandorte in der Schweiz andererseits führen zunehmend zu einer reinen Bewertung des Versuchs nach geltenden Paragraphen anstelle einer ethischen Würdigung mit besonderem Blick auf die Patienten bzw. Probanden. Ein Studienprojekt einer lokalen Ärztegruppe ohne Sponsor kann nicht über denselben Leist wie die globalen MultiCenter-Studien der Pharmariesen geschlagen werden. Im Gegenteil brauchen lokale Kleinprojekte, die für Patienten auch direkte Vorteile mit sich bringen können, die Unterstützung dezentraler, kantonaler Ethikkommissionen. Im Bewilligungsprozess kann die Ethikkommission ihre Erfahrung in das lokale Studienprojekt einfliessen lassen und damit das Qualitätsniveau solcher Versuche heben. Mit dem notwendigen Freiraum versehen, können die kantonalen Ethikkommissionen vor Ort verantwortungsvoll diese Aufgabe wahrnehmen und dazu beitragen, wertvolle Kleinstudien ohne industrielle Sponsoren mach- und finanzierbar zu machen. 